\title{
On the Arbitrariness of the General Solution of an Involutive Partial Differential Equation
}

\author{
Werner M. Seiler ${ }^{\star}$ \\ Centre de recherches mathématiques, Université de Montréal \\ C.P. 6128 Succ. A, Montréal H3C 3J7, Canada \\ Email: seilerw@ere.umontreal.ca
}

\begin{abstract}
The relationship between the strength of a differential equation as introduced by Einstein, its Cartan characters and its Hilbert polynomial is studied. Using the framework of formal theory previous results are extended to non-linear equations of arbitrary order and to over-determined systems. The problem of computing the number of arbitrary functions in the general solution is treated. Finally, the effect of gauge symmetries is considered.
\end{abstract}

PACS: 02.30.J Partial Differential Equations

\section{Introduction}

Usually, it is not possible to construct the general solution of a non-linear partial differential equation. Nevertheless one can often deduce many properties of solutions and/or the solution space without explicitly solving the equation. In this paper, we study several methods for measuring the "richness" or arbitrariness of the solution space. For systems with gauge symmetries, we can refine the question by considering only "physically distinguishable" solutions, i.e. we identify solutions connected by gauge transformations.

To know the arbitrariness of the solution space can be important in many applications. We cite here just two. A typical problem is to check whether or not a solution constructed by some technique is the general solution. We will give a simple criterion for this question using the Hilbert polynomial.

To compute the infinitesimal symmetries of a differential equation requires us to solve the determining system [1,2]. Even if it is not possible to construct the solution explicitly, it is still of considerable interest to know the size of the symmetry group. In the case of the so-called "non-classical method" [3] the determining system is no longer linear and usually the known algorithms to compute the size of the group $[4,5]$ cannot be applied.

\footnotetext{
* Permanent address: Institut für Algorithmen und Kognitive Systeme, Universität Karlsruhe, D-7500 Karlsruhe 1, Germany; email: kg04@dkauni2.bitnet
} 
The notion of strength of a differential equation was introduced by Einstein [6] while searching for a unified field theory. A discussion of similiar ideas appeared already in the correspondence between Cartan and Einstein [7]. Since then it has been applied a few times to physical systems like Maxwell or Einstein equations $[8,9,10,11]$. Later, Sué [12] derived an explicit formula for the strength in terms of the number of equations and "identities".

Cartan developed his theory of involutive systems [14, 13], in which he introduced the Cartan characters, mainly for exterior differential systems but also gave a version for quasi-linear partial differential equations [15]. The characters are related to the number of arbitrary functions in the general solution. It is one of the main goals of this paper to exhibit this relationship in more detail.

The Hilbert polynomial comes originally from algebraic geometry. It describes the number of independent polynomial functions of a given order from a variety into the base field in the coordinates of an embedding space. For differential equations this can be translated into the number of free Taylor coefficients in the general solution. More about this analogy can be found in [16].

Our approach in this paper will be based on an analysis of formal power series solutions. In contrast to earlier attempts (see e.g. [8, 12]) we will make strong use of the formal theory of differential equations [17]. This allows us to compute directly the Cartan characters, the Hilbert polynomial and the strength even for non-linear systems of arbitrary order. Furthermore the results remain valid for over-determined systems.

The paper is organized as follows: Section 2 introduces briefly the notion of a system in involution and its symbol. Section 3 relates the Cartan characters, the Hilbert polynomial and the strength of an involutive system. The next two sections deal with the problem of computing the number of arbitrary functions in the general solution. Section 6 considers the effect of gauge symmetries. Finally, after a section with detailed examples some conclusions are given. Two appendices exhibit some technical lemmata.

\section{Involutive Systems}

Formal theory is based on jet bundle formalism. It is beyond the scope of this paper to give a detailed introduction to the underlying theory. We must refer to the literature [17]. Let $x_{1}, \ldots, x_{n}$ and $u^{1}, \ldots, u^{m}$ be a local coordinate system on a bundle $\mathcal{E}$. We define a differential equation of order $q$ as a fibred submanifold $\mathcal{R}_{q}$ in the jet bundle $J_{q} \mathcal{E}$. Locally, $\mathcal{R}_{q}$ can be described by a system of equations $\Phi^{\tau}\left(x^{i}, u^{\alpha}, p_{\mu}^{\alpha}\right)=0$, where $p_{\mu}^{\alpha}=\partial^{|\mu|} u^{\alpha} / \partial x^{\mu}$.

The prolongation $\mathcal{R}_{q+1} \subset J_{q+1} \mathcal{E}$ is obtained by formally differentiating all equations with respect to the independent variables $x^{i}$. It is well-known that during prolongation integrability conditions can arise, i.e. the dimension of the projected system $\mathcal{R}_{q}^{(1)}=\pi_{q}^{q+1}\left(\mathcal{R}_{q+1}\right)$ is less than the dimension of the original system $\mathcal{R}_{q}$. Such conditions can occur at any prolongation order. A system that does not generate integrability conditions is called formally integrable, because it is possible to construct order by order a formal power series solution. 
A formally integrable system is, however, not yet necessarily involutive. We request further that it has an involutive symbol. The symbol $\mathcal{M}_{q}$ of $\mathcal{R}_{q}$ is a system of linear (algebraic, not differential!) equations in some unknowns $v_{\mu}^{\alpha}$ defined by:

$$
\mathcal{M}_{q}: \sum_{\alpha,|\mu|=q} \frac{\partial \Phi^{\tau}}{\partial p_{\mu}^{\alpha}} v_{\mu}^{\alpha}=0 .
$$

(By abuse of language, we will refer to both the linear system and its solution space as the symbol).

For a quasi-linear equation the symbol is essentially obtained by substituting $v_{\mu}^{\alpha}$ for $p_{\mu}^{\alpha}$ in the highest-order part of the equation. It is a linear system in a vector space $V_{q}$ whose dimension is determined by the number of derivatives of order $q$

$$
\operatorname{dim} V_{q}=m\left(\begin{array}{c}
n+q-1 \\
n-1
\end{array}\right) .
$$

A jet variable $p_{\mu}^{\alpha}$ with multi-index $\mu=\left[i_{1}, \ldots, i_{n}\right]^{2}$ is said to be of class $k$, if $i_{k}$ is the first non-vanishing entry. We order the columns of the symbol $\mathcal{M}_{q}$ by class (highest class first) and compute a row echelon form. We define $\beta_{q}^{(k)}$ as the number of equations where the leading term is of class $k$. The symbol $\mathcal{M}_{q}$ is called involutive, if

$$
\operatorname{rank} \mathcal{M}_{q+1}=\sum_{k=1}^{n} k \beta_{q}^{(k)} .
$$

Associating with each equation of class $k$ its so-called multiplicative variables $x^{1}, \ldots, x^{k}$ and assuming that the symbol is in row echelon form, we see that if we prolong each equation with respect to its multiplicative variables only, we get algebraically independent equations. Equation (3) tells us, that in the case of an involutive symbol no further independent equations exist.

Although this definition appears to be coordinate dependent, one can show that with the exception of certain singular systems every coordinate system yields the same values for the $\beta_{q}^{(k)}$. For lack of space, we can not discuss here the delicate question of this so-called $\delta$-regularity of a coordinate system but refer to the literature [17].

Finally, one should note that any system can be algorithmically completed to an involutive one $[17,18]$. This is ensured by the so-called Cartan-Kuranishi theorem. Thus it poses no real restriction, if we assume from now on that we deal only with involutive systems.

\section{Cartan Characters, Hilbert Polynomial, and Strength}

In the previous section we used the symbol as a criterion for whether a system is in involution or not. The symbol of an involutive system contains, however, much more information on the solution space. The Cartan characters will be our basic tool to extract it.

\footnotetext{
${ }^{2}$ This means $p_{\mu}^{\alpha}=\frac{\partial^{\left(i_{1}+\cdots+i_{n}\right)_{u}^{\alpha}}}{\partial x_{1}^{i_{1}} \cdots \partial x_{n}^{i_{n}}}$.
} 
Definition 1. The Cartan characters $\alpha_{q}^{(k)}$ of the differential equation $\mathcal{R}_{q}$ in $n$ independent and $m$ dependent variables are

$$
\alpha_{q}^{(k)}=m\left(\begin{array}{c}
q+n-k-1 \\
q-1
\end{array}\right)-\beta_{q}^{(k)} \quad k=1, \ldots, n .
$$

$\beta_{q}^{(k)}$ gives the number of principal derivatives of class $k$, i.e. derivatives which can be computed using $\mathcal{R}_{q}$ by solving for them; $\alpha_{q}^{(k)}$ is the number of parametric derivatives of class $k$, i.e. derivatives which must be provided by initial or boundary conditions to specify a unique solution.

An important property of the Cartan characters is that they form a descending sequence [17]:

$$
\alpha_{q}^{(1)} \geq \alpha_{q}^{(2)} \geq \cdots \geq \alpha_{q}^{(n)} .
$$

Definition 2. The Hilbert polynomial $H_{q}(r)$ counts for $r \geq 0$ the number of arbitrary Taylor coefficients of order $q+r$ in the general formal power series solution of the differential equation $\mathcal{R}_{q}$.

Thus, the Hilbert polynomial is given by $H_{q}(r)=\operatorname{dim} \mathcal{M}_{q+r}$, as for a formally integrable system the Taylor coefficients of order $q+r$ can be found as solutions of an inhomogeneous linear system with the same matrix as the prolonged symbol $\mathcal{M}_{q+r}$. Note that we consider only formal power series. No statement about the convergence of the series is made.

In Appendix A the following formula for the dimension of a prolonged symbol is derived using a recursion relation for the Cartan characters:

$$
H_{q}(r)=\operatorname{dim} \mathcal{M}_{q+r}=\sum_{k=1}^{n}\left(\begin{array}{c}
r+k-1 \\
r
\end{array}\right) \alpha_{q}^{(k)} .
$$

To express the coefficients of $H_{q}(r)$ in terms of the Cartan characters, we must write the binomial coefficients explicitly as polynomials in $r$. This is most easily done using the symmetric $q$-products defined in Appendix B. With (40) we find

Theorem 3. The Hilbert polynomial of an involutive system $\mathcal{R}_{q}$ with Cartan characters $\alpha_{q}^{(k)}$ is given by

$$
H_{q}(r)=\sum_{i=0}^{n-1}\left(\sum_{k=i}^{n-1} \frac{\alpha_{q}^{(k+1)}}{k !} s_{k-i}^{(k)}(0)\right) r^{i} .
$$

There is obviously a one-to-one correspondence between the coefficients of the Hilbert polynomial and the Cartan characters. We omit here the explicit expressions for the $\alpha_{q}^{(k)}$ in terms of the coefficients of $H_{q}(r)$.

A system $\mathcal{R}_{q}$ with vanishing Cartan characters is said to be of finite type as there are no free Taylor coefficients of order $q$ or higher. Hence such a system has a finite dimensional solution space parametrized by $\operatorname{dim} \mathcal{R}_{q}$ parameters. We will assume in what follows that at least one character is non-zero. 
To define the strength of a differential equation, we introduce following Einstein [6]

$$
Z_{q}(r)=H_{q}(r) /\left[\begin{array}{c}
n \\
q+r
\end{array}\right]
$$

where we have used the notion $\left[\begin{array}{c}n \\ k\end{array}\right]=\left(\begin{array}{c}n+k-1 \\ k\end{array}\right)$ for the number of Taylor coefficients of order $k$ of a function of $n$ variables (cf. (2)).

$Z_{q}$ is a rational function of $r$. The denominator is of degree $n-1$; the numerator has a degree less than or equal to $n-1 . Z_{q}$ describes the growth of the number of free Taylor coefficients in the general solution relative to a function of $n$ variables. We expand $Z_{q}(r)$ in powers of $1 / r$

$$
Z_{q}(r)=Z_{q}^{(0)}+Z_{q}^{(1)} / r+O\left(1 / r^{2}\right)
$$

Definition 4. We call $Z_{q}^{(0)}$ the compatibility coefficient and $Z_{q}^{(1)}$ the strength of $\mathcal{R}_{q}$. A system with $Z_{q}^{(0)}=0$ is said to be absolutely compatible ${ }^{3}$.

Using (7) and (40), $Z_{q}^{(0)}$ is readily obtained by taking the limit $r \rightarrow \infty$. Similiarly, multiplying (9) by $r$ and taking again the limit yields $Z_{q}^{(1)}$.

Theorem 5. The compatibility coefficient and the strength of an involutive system $\mathcal{R}_{q}$ with Cartan characters $\alpha_{q}^{(k)}$ are given by

$$
\begin{gathered}
Z_{q}^{(0)}=\alpha_{q}^{(n)}, \\
Z_{q}^{(1)}=(n-1)\left(\frac{1}{2} n \alpha_{q}^{(n)}+\alpha_{q}^{(n-1)}\right) .
\end{gathered}
$$

In the next section, we will give an alternative description of the strength, which allows for an easy interpretation of this result.

\section{Arbitrary Functions}

We will try now to arrange the free coefficients of the general formal power series solution into formal series for some arbitrary functions. Again we will not discuss the question of convergence of these series. Although the Cartan-Kähler theorem $[19,17]$ guarantees the existence of an analytic solution for analytic, involutive systems, this does not suffice to conclude that the series for the free functions converge. Furthermore, the number of these functions is not necessarily uniquely defined, as there may exist many different ways to express the general solution by some arbitrary functions.

We make two crucial assumptions to get a unique result:

(i) The general formal power series solution can be constructed order by order, i.e. its principal coefficients of order $q+r$ depend only on the parametric coefficients of order $q+r$ and lower.

\footnotetext{
${ }^{3}$ Einstein introduced the strength only for absolutely compatible systems. It is, however, well-defined for any system.
} 
(ii) The general solution of the system $\mathcal{R}_{q}$ can be written as an algebraic expression, i.e. without derivatives or integrations, of the form

$$
u^{\alpha}(x)=\Psi^{\alpha}\left(x, F_{0}, F_{1}, F_{2}, \ldots, F_{n}\right)
$$

where $F_{k}$ denotes a family of $f_{k}$ functions of $k$ arguments and the $\Psi^{\alpha}$ are some fixed functions. Functions of zero arguments are of course constants.

A solution in closed form requires explicit expressions for the $\Psi^{\alpha}$ and for the arguments of the free functions. A typical example is provided by the wave equation $u_{t t}-u_{x x}=0$ with a general solution of the form $f(x-t)+g(x+t)$, i.e. $\Psi(a, b)=a+b$ and $F_{1}=\{f, g\}, F_{0}=F_{2}=\{\}$.

A function of $k$ variables has $\left[\begin{array}{c}k \\ i\end{array}\right]$ Taylor coefficients of order $i$. Expression (12) for the general solution yields a total of

$$
T_{q}(r)=\sum_{k=1}^{n} f_{k}\left[\begin{array}{c}
k \\
q+r
\end{array}\right]
$$

free Taylor coefficients of order $q+r$. Because of assumption (i) $T_{q}(r)$ must equal $H_{q}(r)$. Expanding both as polynomials in $r$ and equating the coefficients leads to a triangular system of linear equations for the $f_{k}$ which can be easily solved by back-substitution.

The solution of this linear system may, however, contain negative numbers. Indeed we will see in Section 7 that this happens for example in the case of the Maxwell equations in the potential formulation. This means one of the assumptions was incorrect in this case. The next section will study in detail the effect of dropping assumption (ii). We will stick, however, to assumption (i) throughout this paper.

Theorem 6. If there exists a representation of the general solution of the involutive system $\mathcal{R}_{q}$ with Cartan characters $\alpha_{q}^{(k)}$ satisfying both assumptions, then it contains $f_{k}$ free functions of $k$ variables, where the $f_{k}$ are determined by the recursion relation:

$$
\begin{gathered}
f_{n}=\alpha_{q}^{(n)}, \\
f_{k}=\alpha_{q}^{(k)}+\sum_{i=k+1}^{n} \frac{(k-1) !}{(i-1) !}\left(\alpha_{q}^{(i)} s_{i-k}^{(i-1)}(0)-f_{i} s_{i-k}^{(i-1)}(q)\right), \quad 0<k<n .
\end{gathered}
$$

Such a representation can exist only, if the solution of this recursion relation contains only non-negative integers.

We can now express the compatibility coefficient and the strength through the $f_{k}$ instead of the $\alpha_{q}^{(k)}$ :

$$
\begin{gathered}
Z_{q}^{(0)}=f_{n}, \\
Z_{q}^{(1)}=(n-1)\left(\frac{1}{2}(n+2 q) f_{n}+f_{n-1}\right) .
\end{gathered}
$$


Thus a system is absolutely compatible, if its general solution does not contain free functions of $n$ variables. We expect this property from any reasonable system which is not under-determined, as it poses otherwise no real restrictions on at least some of the dependent variables. In physical field equations this is not acceptable. For an absolutely compatible system the strength measures the dominant term in the Hilbert polynomial, the coefficient of $r^{n-2}$.

With the results of Appendix B we can solve the recursion relation (14) explicitly for first-order systems:

$$
\begin{gathered}
f_{n}=\alpha_{1}^{(n)}=m-\beta_{1}^{(n)}, \\
f_{k}=\alpha_{1}^{(k)}-\alpha_{1}^{(k+1)}=\beta_{1}^{(k+1)}-\beta_{1}^{(k)} .
\end{gathered}
$$

The proof is by descending induction. The case $k=n-1$ is obvious from (42). If we assume that (16) holds for $k+1$, we get for $k$ that

$$
\begin{aligned}
f_{k}= & \alpha_{1}^{(k)}-\alpha_{1}^{(k+1)}+ \\
& (k-1) ! \sum_{i=k+2}^{n-1} i ! \alpha_{1}^{(i+1)}\left(i s_{i-k}^{(i-1)}(1)+s_{i-k+1}^{(i)}(0)-s_{i-k+1}^{(i)}(1)\right) .
\end{aligned}
$$

But (44) implies that the expression in the bracket vanishes for all $i, k$. It follows from (5) that the $f_{k}$ are always non-negative. For first-order system it is thus always possible to construct an algebraic expression for the general solution.

It is perhaps interesting to compare (16) with the Cauchy-Kowalevsky theorem. It applies to so-called normal first-order systems, i.e. the number of equations is equal to the number of dependent variables and by a change of the independent coordinates we can always achieve that all equations are of class $n$. Thus $\beta_{1}^{(n)}=m, \beta_{1}^{(n-1)}=\cdots=\beta_{1}^{(1)}=0$. This leads to the well-known result $f_{n}=f_{n-2}=\cdots=f_{1}=0, f_{n-1}=m$.

\section{Integrals in the General Solution}

We have seen in the previous section that it may not always be possible to express the general solution algebraically. But even if Theorem 6 yields only non-negative values for the $f_{k}$, it is interesting to study the effect of more general expressions than (12), as many solution methods for differential equations lead for example to integral representations. If we drop assumption (ii), further terms appear in the definition of $T_{q}(r)^{4}$

$$
T_{q}(r)=\sum_{j \in J} \sum_{k=1}^{n} f_{k, j}\left[\begin{array}{c}
k \\
q+r+j
\end{array}\right] .
$$

\footnotetext{
${ }^{4}$ In order to get a well-defined expression for $j<-q$, we will adopt the convention $\left[\begin{array}{l}n \\ k\end{array}\right]=0$ for $k<0$.
} 
$J$ is some finite set of integers. $j=0$ gives the algebraic case. Positive values of $j$ correspond to derivatives; they occur for example in gauge systems. Negative values denote integrals.

The equality $H_{q}(r)=T_{q}(r)$ leads now to the following linear system of $n$ diophantine equations

$$
\sum_{j \in J} \sum_{k=i}^{n-1} \frac{n !}{k !} s_{k-i}^{(k)}(q+j) f_{k+1, j}=n ! h_{i}, \quad i=0, \ldots, n-1
$$

where $h_{i}$ denotes the coefficients of the Hilbert polynomial. We have multiplied each equation by $n$ ! to avoid rational coefficients. All coefficients including the right hand side are now non-negative integers.

If $|J|>1$, then the system is under-determined, i.e. the number of arbitrary functions is no longer uniquely defined. Even if we disregard how a function enters the general solution and consider only the total number $\tilde{f}_{k}=\sum_{j \in J} f_{k, j}$ of functions with $k$ variables, we get in general no unique results, as we will show later in this section. There exists, however, one invariant:

Theorem 7. Let $\alpha_{q}^{\left(k_{0}\right)}$ be the highest non-vanishing Cartan character of the involutive equation $\mathcal{R}_{q}$. Then every non-negative solution $\left\{f_{k, j}\right\}$ of the diophantine system (18) satisfies

$$
\begin{gathered}
f_{k, j}=0, \quad \forall j \in J, k>k_{0}, \\
\tilde{f}_{k_{0}}=\sum_{j \in J} f_{k_{0}, j}=\alpha_{q}^{\left(k_{0}\right)} .
\end{gathered}
$$

The proof is simple, as under the assumption of the theorem $H_{q}(r)$ is a polynomial of degree $k_{0}-1$. Setting $h_{i}=0$ for $i=k_{0}, \ldots, n-1$ leads consecutively to the equations $\tilde{f}_{n}=0, \ldots, \tilde{f}_{k_{0}+1}=0$. Since only non-negative solutions are allowed, this entails (19). The next order provides (20).

Note that it is essential for this theorem that we still maintain assumption (i). Otherwise the ansatz $H_{q}(r)=T_{q}(r)$ is no longer correct and we can draw no conclusions. We call $\alpha_{q}^{\left(k_{0}\right)}$ the degré d'arbitraire (or index of generality) of the differential equation $\mathcal{R}_{q}$. $k_{0}$ is sometimes called Cartan genus of $\mathcal{R}_{q}$.

The condition that all $f_{k, j}$ must be non-negative implies that only a finite number of solutions can exist, as the right hand sides in (18) can be seen as bounds on weighted sums of the $f_{k, j}$. One can readily design an algorithm which generates for any given set $J$ all non-negative solutions of (18).

Since the number of solutions may be very large, it is nevertheless interesting to study several ways to construct special solutions. The easiest possibility is to set $J=\left\{j_{0}\right\}$. The condition $H_{q}(r)=T_{q}(r)$ leads then to a well-determined linear system of equations for the $f_{k, j_{0}}$. Its solution is given by recursion relation (14), if we substitute in the second symmetric $q$-product the argument $q$ by $q+j_{0}$.

For two special values of $j_{0}$ this modified recursion relation can easily be solved. Setting $j_{0}=-q$ gives at once $f_{k,-q}=\alpha_{q}^{(k)}$ for $k=1, \ldots, n$. Choosing 
$j_{0}=1-q$, we find a solution with $(q-1)$-dimensional integrals generalizing (16): $f_{n, 1-q}=\alpha_{q}^{(n)}, f_{k, 1-q}=\alpha_{q}^{(k)}-\alpha_{q}^{(k+1)}$. Again (5) implies that it is a non-negative solution. Note that these two solutions yield the same values for the $\tilde{f}_{k}$ only if $\alpha_{q}^{(n)}=\cdots=\alpha_{q}^{(2)}=0$.

The first one corresponds to the representation of the general solution used in the already above mentioned Cartan-Kähler theorem. This existence and uniqueness theorem is proven by considering a special initial value problem and constructing a solution through a sequence of normal systems for which the CauchyKowalevsky theorem yields the existence and uniqueness of a solution. As a first step, however, the system is rewritten as a first order system. The arbitrary functions appear as initial data. Thus the back-transformation to the original dependent variables makes them to q-dimensional integrals.

Another way to find solutions of (18) is to exploit the relation

$$
\left[\begin{array}{l}
n \\
k
\end{array}\right]=\left[\begin{array}{c}
n \\
k-1
\end{array}\right]+\left[\begin{array}{c}
n-1 \\
k
\end{array}\right]
$$

which is an immediate consequence of the Pascal triangle. If now $f_{k}$ is a negative value in the solution of (14), we can eliminate it through

$$
f_{k+1}\left[\begin{array}{c}
k+1 \\
q+r
\end{array}\right]+f_{k}\left[\begin{array}{c}
k \\
q+r
\end{array}\right]=\left(f_{k+1}+f_{k}\right)\left[\begin{array}{c}
k+1 \\
q+r
\end{array}\right]-f_{k}\left[\begin{array}{c}
k+1 \\
q+r-1
\end{array}\right],
$$

i.e., we "convert" some functions of $k+1$ variables into integrals of functions of $k$ variables! But this may result in a negative value for the new $f_{k+1}$. Then we can apply the same trick again, if $k+1<n$. But if $f_{n}$ becomes negative, the procedure has failed. Thus this method will only work, if $\sum_{i=k}^{n} f_{i}>0$. It can be generalized by iteration of (21) to include higher-dimensional integrals (see [11]).

Finally, we consider the problem mentioned in the introduction of how to decide whether a solution constructed by some technique is the general solution. Under assumption (i) that the corresponding power series can be constructed order by order, we have simply to count the free functions and to compare the resulting $T_{q}(r)$ with the Hilbert polynomial $H_{q}(r)$ of the differential equation. Only for a general solution both polynomials are identical.

\section{Gauge Systems}

In gauge theories one identifies solutions connected by a gauge transformation, as they lead to the same values for the physical observables. Thus one is not interested in the total number of free functions but only in the number of "physical distinguishable" ones. We will consider gauge relations of the following form:

$$
\begin{gathered}
\bar{x}^{i}=\Omega^{i}\left(x^{j}\right) \\
\bar{u}^{\alpha}=\Lambda^{\alpha}\left(x^{i}, u^{\beta}, \lambda_{a}^{(0)}(x), \partial \lambda_{a}^{(1)}(x), \ldots, \partial^{p} \lambda_{a}^{(p)}(x)\right),
\end{gathered}
$$


i.e., $u$ and $\bar{u}$ are related by a gauge transformation $\Lambda$ depending on $\gamma_{0}$ gauge functions $\lambda_{a}^{(0)}(x)$ entering directly, $\gamma_{1}$ gauge functions $\lambda_{a}^{(1)}(x)$ entering through their first derivatives etc ${ }^{5}$.

We assume that all derivatives of order $l$ of $\lambda_{a}^{(l)}(x)$ do explicitly occur in (23) and that all gauge functions depend on $n$ variables. The only reason for these assumptions is simplicity. The same analysis can be readily performed for more general situations but leads to much uglier results.

To derive now the number of free functions, we can no longer simply equate (7) and (13) but must take into account the gauge functions. The gauge relation (23) implies that at order $q+r$

$$
G_{q}(r)=\sum_{l=0}^{p} \gamma_{l}\left[\begin{array}{c}
n \\
q+r+l
\end{array}\right]
$$

Taylor coefficients can be arbitrarily set by a gauge fixing. Thus we must now solve the equation $H_{q}(r)-G_{q}(r)=T_{q}(r)$ which yields for the numbers $p_{k}$ of physical relevant free functions of $k$ variables the following modified form of Theorem 6:

Theorem 8. Let the involutive system $\mathcal{R}_{q}$ with Cartan characters $\alpha_{q}^{(k)}$ be invariant under the gauge transformation (23). If the "physical solution space" can be represented in a form satisfying assumptions (i) and (ii), then it is spanned by $p_{k}=f_{k}-g_{k}$ free functions of $k$ variables, where the $f_{k}$ are given by Theorem 6 and the $g_{k}$ are determined by the recursion relation:

$$
\begin{gathered}
g_{n}=\sum_{l=0}^{p} \gamma_{l}, \\
g_{k}=\frac{(k-1) !}{(n-1) !} \sum_{l=0}^{p} \gamma_{l} s_{n-k}^{(n-1)}(q+l)- \\
\sum_{i=k+1}^{n} \frac{(k-1) !}{(i-1) !} g_{i} s_{i-k}^{(i-1)}(q) .
\end{gathered}
$$

Such a representation can exist only, if all $p_{k}$ are non-negative integers.

If some of the $p_{k}$ are negative, we need probably integrals to span the physical solution space. This can again be done with the methods outlined in Section 5. If we apply, however, the first one, i.e. $J=\left\{j_{0}\right\}$, we must substitute in (25) in the argument of the second symmetric $q$-product $q$ by $q+j_{0}$.

The definition of the strength should also be changed to reflect the effect of the gauge symmetry: $Z_{q}(r)=\left(H_{q}(r)-G_{q}(r)\right) /\left[\begin{array}{c}n \\ q+r\end{array}\right]$. This is most easily done by substituting $p_{k}$ for $f_{k}$ in (15). Thus free functions of $n$ variables are now allowed in an absolutely compatible system provided they are gauge functions, as these are physically not observable.

${ }^{5}$ Obviously finite-dimensional symmetry groups are of no interest here. 
Independently of any specific expansion of the Hilbert polynomial, we can use the following modified form of Theorem 5 :

Theorem 9. If the involutive system $\mathcal{R}_{q}$ with Cartan characters $\alpha_{q}^{(k)}$ is invariant under the gauge transformation (23), then its gauge corrected compatibility coefficient and strength are given by

$$
\begin{gathered}
Z_{q}^{(0)}=\alpha_{q}^{(n)}-\sum_{l=0}^{p} \gamma_{l} \\
Z_{q}^{(1)}=(n-1)\left(\frac{1}{2} n \alpha_{q}^{(n)}+\alpha_{q}^{(n-1)}-\sum_{l=0}^{p}\left(\frac{1}{2} n+q+l\right) \gamma_{l}\right) .
\end{gathered}
$$

\section{Examples}

Many of the effects discussed in the previous sections are nicely demonstrated by the Maxwell equations. There exist two different formulations: Using the vector potential $A_{\mu}$ they form a second-order system with $n=4$ independent and $m=4$ dependent variables

$$
\mathcal{R}_{2}:\left\{\partial_{\mu} \partial_{\mu} A_{\nu}-\partial_{\mu} \partial_{\nu} A_{\mu}=0 ;\right.
$$

using the field strength

$$
F_{\mu \nu}=\partial_{\mu} A_{\nu}-\partial_{\nu} A_{\mu}
$$

they form a first-order system with $m=6$ dependent variables

$$
\mathcal{R}_{1}:\left\{\begin{array}{l}
\partial_{\mu} F_{\mu \nu}=0 \\
\partial_{[\rho} F_{\mu \nu]}=0
\end{array}\right.
$$

(The signature of the metric has no effect on the formal analysis.)

We readily obtain $\beta_{2}^{(4)}=3, \beta_{2}^{(3)}=1$ for $(27)$ and $\beta_{1}^{(4)}=6, \beta_{1}^{(3)}=2$ for (29). All other $\beta_{q}^{(k)}$ vanish. This yields for the equations in field strength formulation $\alpha_{1}^{(1)}=\alpha_{1}^{(2)}=6, \alpha_{1}^{(3)}=4$ and $f_{1}=f_{4}=0, f_{2}=2, f_{3}=4$. Thus we can write the general solution as an algebraic expression with four functions of three variables and two functions of two variables. For the potential formulation (27) we obtain however $\alpha_{2}^{(1)}=16, \alpha_{2}^{(2)}=12, \alpha_{2}^{(3)}=7, \alpha_{2}^{(4)}=1$ and this leads to $f_{1}=f_{2}=-1, f_{3}=5, f_{4}=1$. Consequently no algebraic expression for the general solution exists.

However, if we also allow one-dimensional integrals, we can find the following solution of the condition $\mathrm{H}_{2}(r)=T_{2}(r)$ :

$$
\begin{gathered}
f_{1,0}=f_{2,0}=f_{3,0}=f_{4,0}=1, \\
f_{2,-1}=2, f_{3,-1}=4 .
\end{gathered}
$$

But this is an obvious consequence of the definition of the field strength: The general solution of (28) considered as a system of differential equations for $A_{\mu}$ 
contains the four functions entering algebraically in (30). The other six functions are just the free functions occuring in the general solution of Equation (29).

These four functions reflect the gauge freedom in the potential formulation. It is well-known that the field strength remains unchanged under the gauge transformation $A_{\mu} \rightarrow A_{\mu}+\partial_{\mu} \lambda(x)$. Indeed, Theorem 8 yields with $\gamma_{1}=1$ the gauge corrections $g_{1}=g_{2}=g_{3}=g_{4}=1$. Subtracting them leads to the same result as for the field strength formulation.

We can also try the second method of Section 5. Applying (22) two times yields

$$
f_{2,-1}=1, f_{3,-1}=2, f_{3,0}=3, f_{4,0}=1 .
$$

Since we now have $f_{1,0}=f_{2,0}=0$, the gauge corrections according to Theorem 8 gives $p_{1,0}=p_{2,0}=-1$. Applying (22) again two times, we arrive at the same result as above, namely four functions of three and two of two variables all entering through integrals.

Using only one-dimensional integrals, i.e. setting $J=\{-1\}$ in (18), results in the following solution:

$$
f_{1,-1}=4, f_{2,-1}=5, f_{3,-1}=6, f_{4,-1}=1 .
$$

The gauge correction must now also be computed with $j=-1$. Theorem 8 yields $g_{1}=4, g_{2}=3, g_{3}=2, g_{4}=1$ and subtraction leads once again to the same final result.

To show that other results are also possible, we consider finally $J=\{-2\}$. After gauge corrections we end up with

$$
f_{1,-2}=f_{2,-2}=6, f_{3,-2}=4 .
$$

The same result is obtained in the field strength formulation setting $J=\{-1\}$. Setting $J=\{0,-1\}$ in this formulation leads to 80 different non-negative solutions of the diophantine system (18) with 25 different sets of values for the $\tilde{f}_{k}$.

Using either the field strength or the potential formulation and Theorem 5 and Theorem 9, respectively, shows that the Maxwell equations are absolutely compatible and have a strength of 12 . Generally, all Yang-Mills theories are absolutely compatible and the strength of a theory with a $d$-dimensional gauge group in an $n$-dimensional space-time is $\left(2 n^{2}-6 n+4\right) d$.

\section{Conclusions}

We see from Theorem 3, that the Hilbert polynomial and the Cartan characters contain the same information. There is a one-to-one correspondence between them. The strength (plus the compatibility coefficient) is a weaker measure, as it takes only two characters into account. The degré d'arbitraire as introduced in Section 5 is stronger. Firstly, because in general it takes more characters into account, namely all vanishing ones. In the case of an absolutely compatible system it uses therefore at least two. Secondly, because Theorem 7 tells us that 
it provides the only true invariant, i.e. the number of free functions of maximal number of arguments.

Sué's [12] conclusion of the opposite is somewhat misleading, because he assumed that we do not know which $k_{0}$ corresponds to the degré d'arbitraire. This is, however, an unrealistic situation. The reason to consider only this one Cartan character lies of course in Theorem 7 and not in a lack of information. Furthermore, he compares a gauge corrected strength with an uncorrected degré d'arbitraire.

Generally, we can conclude that it does not make much sense to measure the arbitrariness of the solution space by the number of arbitrary functions. We have seen that this approach does not lead to unique and thus comparable results, as they depend on the chosen representation of the general solution. We encounter here the typical problem of measuring an infinite-dimensional space by some finite number. The best measure is probably provided by the Hilbert polynomial, as it counts simply the free Taylor coefficients independently of any representation of the solution space.

If we compare our approach with earlier ones, especially by Hoenselaers [8] and Sué [12], we note that their ansatz to compute the Taylor coefficients from the number of equations and identities leads to problems for over-determined systems, as these require that an infinite number of identities be taken into account (thus they are not "systems of type (D)" as introduced by Sué).

The trivial example $u_{x}=0, u_{y}=0$ may illustrate this point. It has an infinite sequence of identities of the form $u_{x y}=u_{y x}$ etc., which must all be counted. This can be highly non-trivial for more complicated systems. Equation (6) performs this counting automatically. It is at this point that involution turns out to be crucial.

Identities can be recognized by a rank deficit in the symbol. We must distinguish two cases. If the corresponding equations in the full system are functionally independent, then an integrability condition has occured. This cannot happen in an involutive system. Otherwise (e.g. in case of the Noether identity for the Maxwell equations), the identities are related to so-called compatibility conditions. We do not have to consider them, as they drop out automatically during the construction of the row echelon form of the symbol.

The occurance of negative values for some $f_{k}$ for the Maxwell equations in the potential formulation has already puzzled several authors [8, 10, 11, 12]. Matthews suspected a connection with gauge symmetries and conjectured that negative values correspond to gauge functions. But as we have seen, gauge corrections come from an a posteriori identification of some solutions. They can only be performed after the $f_{k}$ have been computed.

Integrals have implicitly been used by many authors, as they have worked with functions of different "differentiation orders" $j$. However, these orders were mostly assigned ad hoc or by physical considerations and their relation to integrals were never clearly pointed out.

There exist other approaches to computing the number of arbitrary functions in the general solution. One uses Janet-Riquier theory [20,4] to construct initial value problems. The arbitrariness is now measured in the amount of necessary 
initial data. Since involution depends in this theory also on the chosen ordering for the derivatives and since different orderings may lead to different initial value problems, one can also obtain different expansions of the Hilbert polynomial in this approach.

Finally we want to comment on Sué's remark, that the number of free Taylor coefficients is well-defined only for quasi-linear systems. This is surely not correct! After prolongation every system becomes quasi-linear, thus problems can arise only for the coefficients of order less than $q$ for a differential equation $\mathcal{R}_{q}$. Our starting assumption that the equation defines a fibred submanifold ensures that locally we can always solve for $\operatorname{dim} \mathcal{R}_{q}$ derivatives and this provides the needed distinction between principal and parametric derivatives. But even if we consider more general equations defining algebraic varieties, we can perform the same operation on each component of the variety. The number of principal derivatives may vary from component to component but on each component it is a constant, as it is equal to its codimension.

\section{Acknowledgements}

The author wishes to thank J.F. Pommaret for many helpful discussions and P. Winternitz and the CRM for their hospitality. He is supported by Studienstiftung des deutschen Volkes.

\section{A Prolonged Symbols}

To calculate dimensions or ranks of prolonged symbols, we need formulae for their characters. For this purpose we use the important fact that if $\mathcal{M}_{q}$ is involutive, then all its prolongations $\mathcal{M}_{q+r}$ are involutive, too [17].

Lemma 10. If $\mathcal{M}_{q}$ is an involutive symbol with characters $\alpha_{q}^{(k)}$ and $\beta_{q}^{(k)}$, then the characters of its prolongation $\mathcal{M}_{q+r}$ are given by

$$
\begin{aligned}
\alpha_{q+r}^{(k)} & =\sum_{i=k}^{n}\left(\begin{array}{c}
r+i-k-1 \\
r-1
\end{array}\right) \alpha_{q}^{(i)}, \\
\beta_{q+r}^{(k)} & =\sum_{i=k}^{n}\left(\begin{array}{c}
r+i-k-1 \\
r-1
\end{array}\right) \beta_{q}^{(i)} .
\end{aligned}
$$

It suffices to prove (35), as (34) is then an immediate consequence of (4). We use an induction over $r$. By prolonging an equation of class $k$ or higher with respect to $x^{k}$, we get an equation of class $k$. This yields $\beta_{q+1}^{(k)}=\beta_{q}^{(k)}+\cdots+\beta_{q}^{(n)}$ and we have proven (35) for $r=1$. Note that it is crucial here that $\mathcal{M}_{q}$ is involutive, because only then all independent equations in $\mathcal{M}_{q+1}$ are obtained by prolonging each equation in $\mathcal{M}_{q}$ with respect to its multiplicative variables only (cf. Section 2). 
Let us now assume that (35) holds for $r-1$. Using the formula for $r=1$, we have $\beta_{q+r}^{(k)}=\beta_{q+r-1}^{(k)}+\cdots+\beta_{q+r-1}^{(n)}$. Well-known properties of binomial coefficients yield

$$
\beta_{q+r}^{(k)}=\sum_{i=k}^{n} \sum_{l=k}^{i}\left(\begin{array}{c}
r+l-k-2 \\
r-2
\end{array}\right) \beta_{q}^{(i)}=\sum_{i=k}^{n}\left(\begin{array}{c}
r+i-k-1 \\
r-1
\end{array}\right) \beta_{q}^{(i)} .
$$

Lemma 10 allows us to compute easily the rank and the dimension of a prolonged symbol $\mathcal{M}_{q+r}$. By definition we have rank $\mathcal{M}_{q+r}=\sum_{k=1}^{n} \beta_{q+r}^{(k)}$ and $\operatorname{dim} \mathcal{M}_{q+r}=\sum_{k=1}^{n} \alpha_{q+r}^{(k)}$. Substituting (34) and (35) yields

$$
\begin{aligned}
\operatorname{rank} \mathcal{M}_{q+r} & =\sum_{k=1}^{n}\left(\begin{array}{c}
r+k-1 \\
r
\end{array}\right) \beta_{q}^{(k)}, \\
\operatorname{dim} \mathcal{M}_{q+r} & =\sum_{k=1}^{n}\left(\begin{array}{c}
r+k-1 \\
r
\end{array}\right) \alpha_{q}^{(k)} .
\end{aligned}
$$

\section{B Symmetric $q$-Products}

Definition 11. The symmetric q-product $s_{k}^{(n)}(q)$ is defined for non-negative integers $n, k, q$ with $n \geq k$ by

$$
s_{k}^{(n)}(q)=\left\{\begin{array}{cl}
1 & \text { for } k=0, \\
\sigma_{k}^{(n)}(q+1, q+2, \ldots, q+n) & \text { for } 0<k \leq n,
\end{array}\right.
$$

where we denote by $\sigma_{k}^{(n)}$ the elementary symmetric polynomial of degree $k$ in $n$ unknowns.

These products arise in the expansion of binomial coefficients as polynomials

$$
\left(\begin{array}{c}
q+r+n \\
q+r
\end{array}\right)=\frac{1}{n !} \sum_{i=0}^{n} s_{n-i}^{(n)}(q) r^{i}
$$

The following lemma collects some elementary properties.

\section{Lemma 12.}

$$
\begin{gathered}
s_{n}^{(n)}(q)=(q+n) ! / q !, \\
s_{1}^{(n)}(q)=\frac{1}{2}\left(n^{2}+(2 q+1) n\right), \\
s_{k}^{(n)}(q)=s_{k}^{(n-1)}(q)+(q+n) s_{k-1}^{(n-1)}(q), \\
s_{n-k+1}^{(n)}(1)=n s_{n-k}^{(n-1)}(1)+s_{n-k+1}^{(n)}(0) .
\end{gathered}
$$


$(41,42)$ follow immediately from Definition 11 . (43) stems from a similiar property of symmetric polynomials.

For $k=1$ (44) is an obvious consequence of (41). To proceed from $k-1$ to $k$ we use again an induction. For $n=k$ the proposition follows from (42). To go from $n$ to $n+1$ we apply (43) to each term and order the result as follows

$$
\begin{aligned}
(n+1) s_{n-k+1}^{(n)}(1)+ & s_{n-k+2}^{(n+1)}(0)-s_{n-k+2}^{(n+1)}(1)= \\
(n+1) & {\left[n s_{n-k}^{(n-1)}(1)+s_{n-k+1}^{(n)}(0)-s_{n-k+1}^{(n)}(1)\right]+} \\
& {\left[n s_{n-(k-1)}^{(n-1)}(1)+s_{n-(k-1)+1}^{(n)}(0)-s_{n-(k-1)+1}^{(n)}(1)\right]+} \\
& {\left[s_{n-k+1}^{(n-1)}(1)+(n+1) s_{n-k}^{(n-1)}(1)-s_{n-k+1}^{(n)}(1)\right] . }
\end{aligned}
$$

The first bracket vanishes because we assume that (44) holds for $n$; the second bracket is our proposition for $k-1$. The last one is just (43).

\section{References}

1. G.W. Bluman and S. Kumei. Symmetries and Differential Equations. Applied Mathematical Sciences 81. Springer-Verlag, New York, 1989.

2. P.J. Olver. Applications of Lie Groups to Differential Equations. Graduate Texts in Mathematics 107. Springer-Verlag, New York, 1986.

3. G.W. Bluman and J.D. Cole. The general similarity solution of the heat equation. J. Math. Mech., 18:1025-1042, 1969.

4. G.J. Reid. Algorithms for reducing a system of PDEs to standard form, determining the dimension of its solution space and calculating its Taylor series solution. Eur. J. Appl. Math., 2:293-318, 1991.

5. F. Schwarz. An algorithm for determining the size of symmetry groups. Comp., 49:95-115, 1992.

6. A. Einstein. The Meaning of Relativity. Princeton University Press, Princeton, 5th edition, 1955.

7. E. Cartan and A. Einstein. Lettres sur la Parallélisme Absolue 1929-1932, edited by R. Debever. Palais des Académies, Bruxelles, 1979.

8. C. Hoenselaers. The strength of a system of differential equations. Prog. Theor. Phys., 58:1185-1190, 1977.

9. K.H. Mariwalla. Applications of the concept of strength of a system of partial differential equations. J. Math. Phys., 15:468-473, 1974.

10. N.F.J. Matthews. On the strength of Maxwell's equations. J. Math. Phys., 28:810$814,1987$.

11. B.F. Schutz. On the strength of a system of partial differential equations. $J$. Math. Phys., 16:855-856, 1975.

12. M. Sué. Involutive systems of differential equations: Einstein's strength versus Cartan's degré d'arbitraire. J. Math. Phys., 32:392-399, 1991.

13. E. Cartan. Les Systèmes Différentielles Extérieurs et leurs Applications Géométriques. Hermann, Paris, 1945.

14. R.L. Bryant, S.S. Chern, R.B. Gardner, H.L. Goldschmidt, and P.A. Griffiths. Exterior Differential Systems. Mathematical Sciences Research Institute Publications 18. Springer-Verlag, New York, 1991. 
15. E. Cartan. Sur la Théorie des Systèmes en Involution et ses Applications à la Relativité. In Oeuvres Complètes d'Elie Cartan, Partie II, Vol. 2, pages 1199-1230. Gauthier-Villais, Paris, 1953. (Original: Bull. Soc. Math. France 59 (1931) 88118).

16. G. Carrà Ferro and S.V. Duzhin. Differential-algebraic and differential-geometric approach to the study of involutive symbols. Preprint University of Catania, Italy, 1992.

17. J.F. Pommaret. Systems of Partial Differential Equations and Lie Pseudogroups. Gordon\&Breach, London, 1978.

18. J. Schü, W.M. Seiler, and J. Calmet. Algorithmic methods for Lie pseudogroups. In N. Ibragimov and M. Torrisi, editors, Proc. Modern Group Analysis: Advanced Analytical and Computational Methods in Mathematical Physics, Acireale (Italy), 1992. Kluwer, Dordrecht, to appear.

19. H.L. Goldschmidt. Integrability criteria for systems of non-linear partial differential equations. J. Diff. Geom., 1:269-307, 1969.

20. M. Janet. Sur les Systèmes d'Équations aux Dérivées Partielles. J. Math., 3:65$151,1920$. 